\title{
Natural and Artificial Intelligence, Language, Consciousness, Emotion, and Anticipation
}

\author{
Daniel M. Dubois \\ Department of Applied Informatics and Artificial Intelligence, \\ HEC Management School, N1, University of Liege, \\ rue Louvrex 14, B-4000 Liège, Belgium \\ email: Daniel.Dubois@ulg.ac.be -url: http://www.sia.hec.ulg.ac.be \\ and \\ Centre for Hyperincursion and Anticipation in Ordered Systems (CHAOS ASBL), \\ Institute of Mathematics, B37, Grande Traverse 12, B-4000 Liège 1, Belgium \\ url: http://www.ulg.ac.be/mathgen/CHAOS
}

\begin{abstract}
The classical paradigm of the neural brain as the seat of human natural intelligence is too restrictive. This paper defends the idea that the neural ectoderm is the actual brain, based on the development of the human embryo. Indeed, the neural ectoderm includes the neural crest, given by pigment cells in the skin and ganglia of the autonomic nervous system, and the neural tube, given by the brain, the spinal cord, and motor neurons. So the brain is completely integrated in the ectoderm, and cannot work alone. The paper presents fundamental properties of the brain as follows. Firstly, Paul D. MacLean proposed the triune human brain, which consists to three brains in one, following the species evolution, given by the reptilian complex, the limbic system, and the neo-cortex. Secondly, the consciousness and conscious awareness are analysed. Thirdly, the anticipatory unconscious free will and conscious free veto are described in agreement with the experiments of Benjamin Libet. Fourthly, the main section explains the development of the human embryo and shows that the neural ectoderm is the whole neural brain. Fifthly, a conjecture is proposed that the neural brain is completely programmed with scripts written in biological low-level and high-level languages, in a manner similar to the programmed cells by the genetic code. Finally, it is concluded that the proposition of the neural ectoderm as the whole neural brain is a breakthrough in the understanding of the natural intelligence, and also in the future design of robots with artificial intelligence.
\end{abstract}

Keywords: natural intelligence, neural ectoderm, consciousness, artificial intelligence, robot

PACS: 87.19.L-, 87.19.lv, 87.19.1t, 87.85.dd, 07.05.Bx, 07.05.Mh, 87.85.St

\section{INTRODUCTION}

The classical paradigm of the neural brain as the seat of human natural intelligence is too restrictive.

This paper defends the idea that the neural ectoderm is the actual brain, based on the development of the human embryo. Indeed, the neural ectoderm includes the neural crest, given by pigment cells in the skin and ganglia of the autonomic nervous system, and the neural tube, given by the brain, the spinal cord, and motor neurons. So the brain is completely integrated in the ectoderm, and cannot work alone.

The paper presents some fundamental properties of the brain and is organised as follows.

Firstly, Paul D. MacLean introduced the evolutionary triune brain: the reptilian complex, the limbic system, and the neo-cortex. In short, the first brain controls normal involuntary behaviour with a short-term memory, the second brain is the source of emotions, behaviour and long term memory, and the third brain is involved in higher functions such as sensory perception, generation of motor commands, spatial reasoning, and, in humans, language and conscious thought.

Secondly, the consciousness and conscious awareness are analysed. Many of the advances in the mind-brain problem come from a study of defects in the state of consciousness. 
Thirdly, the anticipatory unconscious free will and conscious free veto are described. Benjamin Libet studied, experimentally, how the human conscious awareness emerges in the brain. His experiments reveal a substantial delay before any awareness affects how we view the human mental activities. If unconscious processes precede any conscious awareness, this means that unconscious processes initiate the conscious experiences. Freely voluntary acts are initiated unconsciously before an awareness of wanting to act. Indeed, he measured the response time between the moment the brain of a patient was stimulated and the time the patient became consciously aware of the stimulus. He found a time delay between the unconscious reaction of the patient and their conscious awareness of the stimulus. This seemed to indicate that free will might not exist in humans at all. But Benjamin Libet disagreed because his experiments showed that, if the volunteer was told not to move a finger or to stop moving it, the conscious will of the volunteer may maintain complete control. So, the conscious will may block the performance of act and veto it.

Fourthly, the main section explains the development of the human embryo and shows that the neural ectoderm is the whole neural brain.

Fifthly, a conjecture is proposed that the neural brain is completely programmed with scripts written in biological low-level and high-level languages, in a manner similar to the programmed cells by the genetic code.

Finally, it is concluded that the proposition of the neural ectoderm as the whole neural brain is a breakthrough in the understanding of the natural intelligence, and also in the future design of robots with artificial intelligence.

\section{THE TRIUNE HUMAN BRAIN}

Paul D. MacLean [1] proposed that the human brain consists to three brains in one, given by the reptilian complex, the limbic system, and the neo-cortex. In his evolutionary triune brain theory, he explains brain function through the evolution of existing structures of the human brain.

The reptilian complex, existing since 400 million years, controls normal involuntary behaviour that the conscious mind does not, such as the cardiac and respiratory functions. The reptilian complex is found in all vertebrates and is the most primitive structure of a brain during the species evolution. It is pre-programmed and has only a short-term memory.

The limbic system, existing since 65 million years, which was first introduced by MacLean in 1952, is similar to the brain of the more primitive mammals and is the source of emotions and behaviour. It operates by influencing the autonomic nervous system and acts as a filter to select information to record in his long-term memory. This limbic filter excites the cortex depending on the filter itself linked to the emotional feelings. It is highly interconnected with the nucleus accumbens, the pleasure center of the brain, which plays a role in sexual arousal and the heavy derived from certain recreational drugs. It is involved in reward, placebo effect, pleasure, laugher, addiction, fear and aggression. The emotions triggered by stimuli acting on the limbic system are not under the control of the cortex. The limbic system is tightly connected to the prefrontal cortex including the orbitofrontal cortex that is required for decision making. The limbic brain has some autonomy from the cortex, in blocking any responsiveness of cortical areas, and in anaesthetizing the unpleasant feelings that do not reach the cortex but may stimulate certain areas of the cortex. This is important to note that the communication is thus unidirectional from the limbic system to the cortex, but the right hemisphere of the cortex may block the communication from the limbic system.

The neo-cortex, also known as the cerebral cortex, existing more recently since 3.6 million years, resembles the brain of more recent mammals in that it controls more highly evolved mental capacity such as reason and speech. It is involved in higher functions such as sensory perception, generation of motor commands, spatial reasoning, and, in humans, mind, language, conscious thought and anticipation of acts.

This is important to know the structure of the brain, because this can explain some anomalies in the way of reasoning and free volition of the conscious mind. For example, the decision making to stop any addiction to products, like alcohol, and drugs, like medications, mainly depends of the limbic system, and thus is not controlled by way of rational reasoning of the neo-cortex. Indeed, the limbic system is the central unconscious evaluator of the information entering the brain. The orbitofrontal cortex is the conscious rational complement of the unconsciously working limbic system. The orbitofrontal cortex unconsciously receives the emotional limbic stimulations, but consciously makes them rational for the real world conditions by way of reasoning. So this is clear that the intelligence deals essentially with the unconscious brain.

Next section deals with the conscious and unconscious intelligence of the brain. 


\section{CONSCIOUSNESS AND CONSCIOUS AWARENESS}

Consciousness is seen as the domain of the mind but it exists within the context of sensory input to and actions carried out by the body.

Amongst the conscious awareness of the body, let us cite the five main senses, sight or vision, hearing or audition, taste, smell or olfaction, touch. Others can be added as balance and acceleration, temperature, kinaesthetic sense, pain, direction. It exists also other internal senses, an internal sense that deals with any sense that is normally stimulated from within the body, as respiration, heartbeat, stomach, etc.

The unconscious mind might be defined as that part of the mind which gives rise to a collection of mental phenomena that manifest in a person mind but which the person is not aware of at the time of their occurrence. These phenomena include unconscious feelings, unconscious automatic skills or reactions, unnoticed perceptions, unconscious thoughts, unconscious habits, and learned things that are done without thinking.

An example of an unconscious operation is the phenomenon where a given problem has no immediate solution, then, days later the unconscious brain provides a solution on a silver platter.

Many of the advances in the mind-brain problem come from a study of defects in the state of consciousness.

A first case is given by a damage within the brain causing problems such as blind-sight, can reveal a great deal about consciousness. The effects of blind-sight cause a person to be fully able to interact with an object in the visual field, yet be totally unaware of its existence. They have no conscious awareness of the object but behave as though they can see it.

A second case deals with a patient who was completely blind in the left visual field as a result of brain surgery. Although he could not see any objects on the left of the visual field, he was able to reach out correctly for them when asked to do so. When questioned, the patient said that he was just guessing. This condition is where a person has no conscious awareness of sight yet certain actions provide evidence that visual experiences are happening at an unconscious level.

A third case concerns some patients who suffer severely from seizure, where uncontrolled activity of the brain spreads throughout the brain and across from one hemisphere to another. The split-brain can alleviate the worst of the symptoms. Split-brain is the result when the corpus callosum connecting the two hemispheres of the brain is severed. The split-brain research began in the 1970s, with the pioneers, Michael Gazzaniga and Roger Sperry, who tested the functioning of each hemisphere independently of the other in split-brain patients. In such patients, one half of the brain processes information presented to one half of the visual field. In tests, information learned using one half of the brain alone is not useable by the other half. It is as if the patient has never seen or learned the information. However, the half of the brain that did see or learn the task is fully aware.

Finally, let us give a fourth case of an individual automatism, which is the alien hand syndrome where a person experiences his hand moves as uncontrolled by himself, although the movements display a complex purposive act.

\section{ANTICIPATORY UNCONSCIOUS FREE WILL AND CONSCIOUS FREE VETO}

Benjamin Libet studied, experimentally, how the human conscious awareness emerges in the brain. In his book, Libet [2] describes all his extraordinary discoveries.

In a pioneered work, Benjamin Libet et al in 1983 [3], found a brain signal, the so-called readiness-potential that occurred a fraction of a second before a conscious decision.

His experiments reveal a substantial delay, the mind time, before any awareness affects how we view the human mental activities.

If unconscious processes precede any conscious awareness, this means that unconscious processes initiate the conscious experiences. Freely voluntary acts are initiated unconsciously before an awareness of wanting to act.

Indeed, Benjamin Libet measured the response time between the moment the brain of a patient was stimulated and the time the patient became consciously aware of the stimulus.

He found there was a consistent half-second delay between the unconscious reaction of the patient and their conscious awareness of the stimulus.

Moreover, he continued his work with even more experiments to refine his theories of mind, brain and consciousness.

Those experiments also involved using electrodes to measure the response times of the brain, and he found, for example, that when a volunteer was instructed to move a finger, the brain unconsciously initiated the movement even before the volunteer was aware that the finger had begun moving.

This seemed to indicate that free will might not exist in humans at all. 
Daniel M. Dubois,"Natural and Artificial Intelligence, Language, Consciousness, Emotion, and Anticipation". In: COMPUTING ANTICIPATORY SYSTEMS: CASYS'09 - Ninth International Conference, edited by D.M. Dubois, American Institute of Physics, AIP CP 1303, pp. 236-245, 2010.

But Benjamin Libet disagreed because his experiments showed that, if the volunteer was told not to move a finger or to stop moving it, the conscious will of the volunteer may maintain complete control.

So, the conscious will may block the performance of act and veto it.

These discoveries have profound implications for the nature of free will.

Indeed, after the results of Benjamin Libet, the free will only consists in the possibility to say NO, what is a veto.

In consequence, the brain in an unconscious way creates and launches the future event before the knowledge of this event reaches the conscious part of the brain.

At that moment, the conscious brain can accept or refuse this event.

This means that in these experiments, there is no place for the conscious brain to create and launch future event.

The unconscious brain anticipates the future decision of the conscious brain, but the conscious brain may block this decision.

Many scientists argued that if the decisions are prepared, unconsciously, by the brain, then the feeling of free will must be an illusion.

In this view, it is the brain that makes the decision.

Decision-making may be a process handled to a large extent by unconscious mental activity.

Recently, the researchers from the group of John-Dylan Haynes [4], from the Max Planck Institute for Human Cognitive and Brain Sciences in Leipzig, in collaboration with the Charité University Hospital and the Bernstein Center for Computational Neuroscience in Berlin, has demonstrated in brain scanning images how the brain might unconsciously prepare our decisions.

It was found that the outcome of a decision could be encoded in brain activity of prefrontal and parietal cortex up to 10 seconds before it enters awareness.

This delay presumably reflects the operation of a network of high-level control areas that begin to prepare an upcoming decision long before it enters awareness.

Indeed, a brain scanner is used to investigate what happens in the human brain just before a decision is made.

In this study, volunteers could freely decide if they wanted to press a button with their left or right hand.

They were free to make this decision whenever they wanted, but had to remember at which time they felt they had made up their mind.

The aim of the experiment was to find out what happens in the brain in the period just before the person felt the decision was made.

The researchers found that it was possible to predict from brain signals which option participants would take up to seven seconds before they consciously made their decision.

\section{THE NEURAL ECTODERM IS THE WHOLE NEURAL BRAIN}

This section deals with a description of the first stages of an embryo to show that the brain is integrated to the whole nervous system, and the skin.

Natural intelligence emerges from the wholeness of brain, spinal cord, nervous systems and skin.

The fact to consider that natural intelligence is the property of the sole neural brain is erroneous, because the brain forms an oneness with the spinal cord, sensory-somatic nervous system, autonomic nervous system, and skin.

Already, in a paper written in 1986, I made a research on natural and artificial intelligence in relation to the logic of the living, where I introduced the development of the embryo, and wrote [5]:

- the ectoderm gives rise to the skin, brain and whole nervous system;

- $\quad$ the mesoderm, gives rise to muscles, bones and circulatory system;

- the endoderm, gives rise to the digestive tract and respiratory system.

The ectoderm is the outer layer of the early embryo. It emerges first and forms from the outer layer of germ cells. The ectoderm has three parts:

- external ectoderm,

- the neural crest, and

- neural tube.

The external ectoderm or surface ectoderm forms the skin (only epidermis), along with glands, hair and nails, sensory receptors in epidermis, lens and cornea of the eye, etc. 
Daniel M. Dubois,"Natural and Artificial Intelligence, Language, Consciousness, Emotion, and Anticipation". In: COMPUTING ANTICIPATORY SYSTEMS: CASYS'09 - Ninth International Conference, edited by D.M. Dubois, American Institute of Physics, AIP CP 1303, pp. 236-245, 2010.

The latter two, the neural crest and neural tube, are known as the neural ectoderm or neuroectoderm. Neuroectoderm is the term for ectoderm that leads to the development of the nervous system from this tissue.

The neural ectoderm includes the neural crest, given by pigment cells in the skin, ganglia of the autonomic nervous system, etc, and the neural tube, given by the brain, the spinal cord, motor neurons, etc.

The neural brain is only a part of the neural ectoderm, and the neural ectoderm is the whole neural brain.

The three layers of primitive cell forming the human embryo internal organs produce ectoderm, mesoderm and endoderm, are shown in Fig. 1.

\section{Development of the human embryo}

\begin{tabular}{|c|c|c|c|c|c|c|}
\hline \multicolumn{5}{|c|}{ Ectoderm } & Mesoderm & Endoderm \\
\hline & \multicolumn{4}{|c|}{ Nervous System } & & \\
\hline & Peripheral Nerv & us System (PNS) & Central Nervous & tem (CNS) & & \\
\hline SKIN & $\begin{array}{l}\text { Sensory-Somatic } \\
\text { Nervous System }\end{array}$ & $\begin{array}{c}\text { Autonomic } \\
\text { Nervous System }\end{array}$ & SPINAL CORD & BRAIN & & \\
\hline
\end{tabular}

FIGURE 1. Embryo divides in three layers: ectoderm, mesoderm and endoderm, then the ectoderm divides in SKIN, PNS and CNS, and finally, the PNS divides in S-SNS and ANS, and CNS divides in Spinal Cord and BRAIN.

The skin and the nervous system originate from the same tissue, the ectoderm.

The nervous system is divided into the Peripheral Nervous System (PNS) and the Central Nervous System (CNS).

The PNS consists of sensory neurons running from stimulus receptors that inform the CNS of the stimuli, and of motor neurons running from the CNS that transmit back a response to action effectors, given by the muscles and glands.

The CNS consists of the spinal cord and the brain.

The peripheral nervous system is subdivided into the Sensory-Somatic Nervous System (between the CNS and the external environment) and the Autonomic Nervous System (between the CNS and the internal environment).

The wholeness of the human natural brain, spinal cord and skin, is shown in Fig. 2.

\begin{tabular}{|c|c|c|c|c|c|c|}
\hline \multicolumn{2}{|c|}{ The Wholeness of the Human Natural Brain, Spinal Cord, and Skin } \\
\hline \multicolumn{2}{|c|}{ PNS: } & CNS: & \multicolumn{3}{c|}{ PNS: } \\
\hline Sensory-Somatic Nervous System & & \multicolumn{2}{c|}{ Autonomic Nervous System } \\
\hline External & $\begin{array}{l}\text { Stimulus } \\
\text { receptors }\end{array}$ & $\begin{array}{l}\text { Sensory } \\
\text { neurons }\end{array}$ & $\begin{array}{c}\text { Sensory } \\
\text { neurons }\end{array}$ & $\begin{array}{c}\text { Stimulus } \\
\text { receptors }\end{array}$ & Internal \\
\hline Environment & SKIN & $\begin{array}{c}\text { SPINAL CORD } \\
\text { and } \\
\text { BRAIN }\end{array}$ & $\begin{array}{c}\text { Motor } \\
\text { neurons }\end{array}$ & $\begin{array}{c}\text { Action } \\
\text { effectors }\end{array}$ & Environment \\
\hline
\end{tabular}

FIGURE 2. Diagram of the Peripheral Nervous System (PNS) and Central Nervous System (CNS), in relation with the internal and external environments.

All our conscious awareness of the external environment and all our motor activity, to cope with it, operate through the sensory-somatic division of the PNS.

The sensory-somatic system consists of 12 pairs of cranial nerves and 31 pairs of spinal nerves. 
Daniel M. Dubois,"Natural and Artificial Intelligence, Language, Consciousness, Emotion, and Anticipation". In: COMPUTING ANTICIPATORY SYSTEMS: CASYS'09 - Ninth International Conference, edited by D.M. Dubois, American Institute of Physics, AIP CP 1303, pp. 236-245, 2010.

The 12 pairs of cranial nerves are given as follows: purely sensory Olfactory nerve for smell, purely sensory Optic nerve for vision, mainly motor Oculomotor nerve for eye movements, mainly motor Trochlear for eyeball, mixed (both sensory and motor) Trigeminal for face, mainly motor Abducens for eye, mixed Facial for facial expression, mostly sensory Vestibulocochlear for hearing and balance, mixed Glossopharyngeal for taste, mixed Vagus for vagal nerve, mainly motor Accessory for swallowing, moving head and shoulder, mainly motor Hypoglossal for tongue.

The 31 pairs of spinal nerves are constituted with 8 cervical, 12 thoracic, 5 lumbar, 5 sacral and 1 coccygeal, which control the functions of the rest of the body. These spinal nerves are mixed because they contain both sensory and motor neurons.

Receptors are specialised nerve cells, which are adapted to respond to a stimulus. For example, each of senses has a particular group of receptors, which respond to a stimulus.

The skin is the outer surface of the body and the envelope of the brain.

Already, in a paper written in 1989, I gave a diagram of a system in interactions with its environment via an envelope, and wrote [6]:

The exchange of matter, energy, information, etc... between the system and its environment is via the characteristics of this envelope, that is to say the actions of the system on the environment and the feedback that follows. We would like to stress here on the fact that a description of the environment is very important to understand and explain the behaviour of the system and its evolution in terms of these interactions.

This is via this envelope that the system that is the brain will create one or several peculiar representations of its environment. It will also be a representation of itself and a consciousness.

The entire skin has a lot of sensors, connected to the spinal cord by nerve fibres.

These sensors give information on touched things and the tactile experience on own body as the target.

Experience of touch plays a role in self-consciousness.

Fig. 3 shows the transmission of information of the motor and sensory system from the body to the two hemispheres of the cortex.

\begin{tabular}{|c|c|c|c|c|}
\hline \multirow{2}{*}{$\begin{array}{c}\text { CORTEX } \\
\text { LEFT HEMISPERE } \\
\text { Analytically rational } \\
\text { Words and Languages } \\
\text { Temporal patterns } \\
\text { Past-Present }\end{array}$} & $\stackrel{\leftrightarrow}{\hookrightarrow \rightarrow \rightarrow}$ & $\begin{array}{c}\text { CORPUS } \\
\text { CALLOSUM }\end{array}$ & $\stackrel{\leftrightarrow}{\hookrightarrow \rightarrow \rightarrow}$ & \multirow{2}{*}{$\begin{array}{c}\text { CORTEX } \\
\text { RIGHT HEMISPHERE } \\
\text { Synthetically intuitive } \\
\text { Symbols and Images } \\
\text { Spatial patterns } \\
\text { Present-Future }\end{array}$} \\
\hline & $\leftarrow \leftarrow \leftarrow$ & $\begin{array}{c}\text { LIMBIC SYSTEM } \\
\text { Emotions } \\
\text { Long-term Memory }\end{array}$ & $\longrightarrow$ & \\
\hline \multicolumn{5}{|c|}{$\boldsymbol{\Delta}$} \\
\hline \multicolumn{5}{|c|}{$\begin{array}{c}\text { BRAIN STEM } \\
\text { Motor and Sensory System }\end{array}$} \\
\hline
\end{tabular}

FIGURE 3. Diagram of the transmission of information of the motor and sensory system from the body to the Brainstem (or Brain Stem), the Limbic System and the two Hemispheres of the Cortex.

The human brain stem, which is the posterior part of the brain, adjoining and structurally continuous with the spinal cord, provides the main motor and sensory innervations to the face and neck via the cranial nerves.

This is a vital part of the brain as the nerve connections of the motor and sensory systems from the main part of the brain to the rest of the body pass through the brain stem.

So the brain stem plays a role in the regulation of cardiac and respiratory function.

It also regulates the central nervous system, and is pivotal in regulating the sleep cycle and maintaining consciousness.

The brain stem sends information of the motor and sensory system towards the limbic system.

The limbic system sends more information to the right hemisphere of the cortex than to the left hemisphere.

By the connections of the corpus callosum, the right hemisphere sends more information to the left hemisphere than from the left hemisphere to the right hemisphere.

To locate the stimuli on the surface of the body, the brain has nerve endings on specific points of the skin. 
After the experiments of Roger Sperry [7], to measure these correspondence points on the skin and brain, a piece of skin were removed from the belly of a frog, and another piece in his back, then relocated two pieces inverting. Subsequently, when tickled the frog on its belly, the frog scratched its back. This mapping in the brain is genetic, and the stimuli of nerve endings on the surface of the skin cannot re-organize connections by learning in the central nervous system. Let us cite Roger Sperry [7, p. 75]:

Implicit in this theory is the assumption that in the embryo the cerebral cortex and the lower relay-centers also undergo a differentiation that parallels in miniature that of the body surface. In other words, just as from the skin to the first central connection point in the spinal cord, so from relay to relay and finally to the cortex the central linkages arise on the basis of selective chemical affinities. At each of its ascending levels the nervous system forms a maplike projection of the body surface.

This maplike projection of the body surface, the surface ectoderm, described by Roger Sperry, may be identified today with what is called a homunculus.

The cortical homunculus is a representation of the anatomical divisions of the primary motor cortex and the primary somato-sensory cortex.

This includes the regions of the human brain dealing with the movement and exchange of sense and motor information, like touch sensitivity, cold, heat and pain, of the rest of the body.

The image is given by a disfigured human with disproportionately huge hands, lips, and face in comparison to the rest of the body.

The motor skills and sense nerves found in these parts of the body are represented as being larger on the homunculus.

This relates to neuro-biological phenomena like phantom limb and body integrity identity disorder.

The cortical homunculus is a self-similar representation of the body within the brain.

Moreover, the whole nervous system has the property of self-similarity.

So, this self-similar property leads to a fractal structure of the neural ectoderm.

In my book, the Labyrinth of Intelligence: From the Natural Intelligence to the Fractal Intelligence [8], a generic fractal system with 7 levels is developed for explaining natural intelligence and designing robots with artificial intelligence.

\section{THE PROGRAMMED BRAIN WITH SCRIPTS LANGUAGES}

This section is a first answer about my conjecture [12]:

When a machine will be able to comprehend our language and to dialogue with us, thus an artificial conscious intelligence will naturally emerge in the machine.

This section will also address the question of the difference between the human natural language and computer artificial language.

The concepts of strong and weak artificial intelligence will be finally discussed.

A natural language is a human written or spoken language as opposed to a computer language, which is an artificial language.

The human natural languages are a means of communication between individuals, through a system of words given by arbitrary signs and symbols, but also a means of self-communication permitting the self-reference, and consciousness.

The emergence of languages was due to the necessity to have a means to memorise space-time events.

So to refer to past, present, and future events, the brain needs a repository medium given by a memory.

For manipulating the content of this memory, a language is necessary.

The ability to recall past events is crucial for the development of intelligence, and the capability of making anticipation is only possible with a language.

The words of natural languages show ambiguities depending on syntax and semantics.

The words are defined in a dictionary.

The syntax for associating the words is given by a grammar.

The semantics deals with the meaning of a word, related to its effect on individuals.

An interesting question deals with the difference between the human natural languages and computer artificial languages. 
Daniel M. Dubois,"Natural and Artificial Intelligence, Language, Consciousness, Emotion, and Anticipation". In: COMPUTING ANTICIPATORY SYSTEMS: CASYS'09 - Ninth International Conference, edited by D.M. Dubois, American Institute of Physics, AIP CP 1303, pp. 236-245, 2010.

In computers, all programs are given by an ordered succession of instructions and commands, written in artificial languages, created by man.

The artificial languages are similar to the natural languages except that there is no ambiguity in their meaning.

The programs written in an artificial high-level language must be translated to the computer low-level language, called the machine code, and executable by the microprocessor.

The translator is called a compiler (who translates the whole program at once) or an interpreter (who compiles an instruction at a time).

The original program is called the source code, and the translated executable program is called the object code.

In machine code, any instruction or command, in artificial languages, gives rise to a specific action on the machine (the computer microprocessor), which represents its meaning.

A virtual machine $(\mathrm{VM})$ is a software implementation of a machine that executes programs like a physical machine. This is an efficient, isolated duplicate of a real machine.

Current use includes virtual machines that have no direct correspondence to any real hardware.

Virtual machines are separated into two major categories, based on their use and degree of correspondence to any real machine.

A system virtual machine provides a complete system platform that supports the execution of a complete operating system (OS).

In contrast, a process virtual machine is designed to run a single program, which means that it supports a single process.

In any artificial language, the fundamental instructions correspond to specific commands.

It is important to note that the input and output of data does not depend of the language, but of the devices for read and write the data.

A program is a sequence of instructions, written in an artificial language, that specifies how to perform a computation.

There are computations of mathematical equations, symbolic systems, or translator programs.

Let us remark that natural systems do not deal with real numbers, with an infinite number of decimals, as computers, as well explained by Herbert Simon [9].

In my recent paper [10], I conjecture that the brain is completely programmed, and there are several types of programs. These programs are scripts embedded in the neural brain. These scripts are based on biological low-level and high-level languages.

Recall that all the cells in the body, and in the neurons in the brain, are also programmed by the genetic code, DNA, in a low-level language with four elementary signs

There are translating scripts that can be called interpreter that translate a script to another script.

There are different types of scripts, some scripts are written in neural low-level languages, and there are interpreter scripts that translate a category of script to another category.

For example, we suggest that an unconscious script becomes a translated conscious script by an interpreter.

In conscious mind, the scripts are written in high-level languages embedded in the neural brain.

The natural languages, like French, English, that Human learns, are high-level languages.

Translation is the comprehension of the meaning of a script and the subsequent production of an equivalent script, likewise called a translation that communicates the same message in another language (Kull, 1998 [11]).

The script that is translated is called the source script, and the language that it is translated into is called the target language. The product is called the target script.

The fact to be conscious or not is a physical property of the brain, because this is the result of the execution of a program represented by a script embedded in the neural brain.

The conscious will, when we become aware of it, is the same will as the unconscious will.

The unconscious will is written in a script embedded in the neural brain, and the fact that this unconscious will becomes a conscious will, is just the translation by an interpreter of the unconscious script to a conscious script embedded in the brain. brain.

The concept of virtual machine may be applied in the neural ectoderm, what may be called by virtual neural

For example, the cortical homunculus may be considered as a virtual neural brain, which is a representation of the anatomical divisions of the primary motor cortex and the primary somato-sensory cortex. The cortical homunculus is a self-similar virtual neural brain of the body within the brain.

Let us note that when I refer to the neural brain, in short, I refer to the whole neural brain given by the neural ectoderm, as explained in the receding section.

The language is a key concept in Natural and Artificial Intelligence. 
Daniel M. Dubois,"Natural and Artificial Intelligence, Language, Consciousness, Emotion, and Anticipation". In: COMPUTING ANTICIPATORY SYSTEMS: CASYS'09 - Ninth International Conference, edited by D.M. Dubois, American Institute of Physics, AIP CP 1303, pp. 236-245, 2010.

Normally, Natural Intelligence deals with the intelligence of living systems in general and of Human in particular. This is a class of mental properties of the mind.

But the mind is related to the brain, and the brain alone is not sufficient to give rise to intelligence.

Indeed, the brain is integrated in the human body by the nervous system, and moreover the body is integrated in its terrestrial environment, via its skin.

To study natural intelligence, without the body and the environment, means to study what is called Pure Intelligence. The mind is a collection of various classes of mental properties, and intelligence is such a class.

This means that intelligence is a property of the brain integrated in the biological body as a whole.

So, this means that intelligence depends to its neural support in the brain and body.

But the intelligence and the mind are independent of its neural brain and body. Indeed, the intelligence can work freely on its neural support given by the brain that is, in turn, entirely guided by the intelligence.

The consequence of this strong condition is that intelligence could be created on an adequate artificial physical or biological robot, which defines what is called a strong artificial intelligence.

We arrive so to the concept of Artificial Intelligence. As such, the intelligence, being a property, cannot be called natural and artificial. What is natural is the neural support of the natural intelligence, given by the natural brain and body of a human being, and what is artificial is the neural support of the artificial intelligence, given by an artificial brain and body of a robot being.

Today, artificial intelligence consists of models of natural intelligence simulated in a Turing machine, and so defines what is called weak artificial intelligence.

\section{CONCLUSION}

The classical paradigm of the neural brain as the seat of human natural intelligence is too restrictive.

The brain is only a part of the body of a Human being, and a brain without a body cannot work. The Human being is only a part of the Environment, and a Human being without an environment cannot exists. The environment is all what exists on earth, in the solar system and in the Universe. The development of a Human being is only possible if there is a set of other Human beings, the whole is the Humanity, with Human societies.

This paper defends the idea that the neural ectoderm is the actual brain, based on the development of the human embryo. Indeed, the neural ectoderm includes the neural crest and the neural tube, given by the brain, the spinal cord, and motor neurons. So the neural brain is completely integrated in the neural ectoderm, and cannot work alone.

The paper presented some fundamental properties of the brain.

Firstly, Paul D. MacLean proposed the triune human brain, which consists to three brains in one, following the species evolution, given by the reptilian complex, the limbic system, and the neo-cortex.

Secondly, the consciousness and conscious awareness are analysed.

Thirdly, the anticipatory unconscious free will and conscious free veto are described in agreement with the experiments of Benjamin Libet.

Fourthly, the main section explains the development of the human embryo and shows that the neural ectoderm is the whole neural brain.

Fifthly, a conjecture is proposed that the neural brain is completely programmed with scripts written in biological low-level and high-level languages, in a manner similar to the programmed cells by the genetic code.

This is a first answer to the conjecture I made in a recent paper [12]:

When a machine will be able to comprehend our language and to dialogue with us, thus an artificial conscious intelligence will naturally emerge in the machine.

Finally, let us conclude in saying that the proposition of the neural ectoderm as the whole neural brain is a breakthrough in the understanding of the natural intelligence, and also in the future design of robots with artificial intelligence.

The ideas presented in this short communication are a starting point to future research and development in view of giving a clear definition of what is natural intelligence and what is consciousness and unconsciousness.

The forthcoming research will deal with a continuation of the proposition of a fractal hologram structure of the whole neural brain, given by the neural ectoderm in natural human intelligence.

This will give a new impulse for designing a robot with an artificial intelligence based on a fractal hologram as initiated in my book [8]: The labyrinth of intelligence: from the natural intelligence to the fractal intelligence. 


\section{REFERENCES}

1. MacLean, Paul D. (1990). The triune brain in evolution: role in paleocerebral functions. New York: Plenum Press.

2. Libet, Benjamin (2004), Mind Time. The Temporal Factor in Consciousness, Harvard University Press, Cambridge, London.

3. Libet, Benjamin, Gleason, C. A., Wright, E. W., \& Pearl, D. K. (1983), Time of conscious intention to act in relation to onset of cerebral activity (readiness-potential). The unconscious initiation of a freely voluntary act, Brain, 106, 623-642.

4. Soon, Chun Siong; Brass, Marcel; Heinze, Hans-Jochen \& Haynes, John-Dylan (2008), Unconscious determinants of free decisions in the human brain. Nature Neuroscience. Volume 11, Number 5, pp. 543-545.

5. Daniel Dubois (1986), L'intelligence artificielle et la logique du vivant, CYBERNETICA, Vol. XXIX, N 3, pp. 175-192.

6. Daniel M. Dubois (1989), Un modèle fractal des systèmes intelligents - A fractal model of intelligent systems, Actes du 1er Congrès Européen de Systémique, Lausanne, 3-5 octobre 1989, AFCET, Tome II, pp. 660-675.

7. Sperry, Roger W. (1959). The Growth of Nerve Circuits, Scientific American, Vol. 201, Nº 5.

8. Dubois, Daniel (1990). LE LABYRINTHE DE L'INTELLIGENCE. De l'intelligence naturelle à l'intelligence fractale. InterEditions (Paris) / ACADEMIA (Louvain-la-Neuve), $332 \mathrm{p}$.

9. Simon, Herbert A. (1980). Computers - Non-numerical computation. Proc. Natl. Acad. Sci. USA, Vol. 77, No. 11, pp. 62646268, November 1980

10. Dubois, Daniel M. (2010). Breakthrough in the Human Decision Making Based on an Unconscious Origin of Free Will, ACTA SYSTEMICA, Volume X, No. 1, pp. 13-18, ISSN 1813-4769

11. Kull, Kalevi (1998). Organism as a Self-reading Text: Anticipation and Semiosis, International Journal of Computing Anticipatory Systems 1, 93-104.

12. Dubois, Daniel M. (2008). New Trends in Computing Anticipatory Systems: Emergence of Artificial Conscious Intelligence with Machine Learning Natural Language. In COMPUTING ANTICIPATORY SYSTEMS: CASYS'07 - Eighth International Conference, Edited by D. M. Dubois, Published by American Institute of Physics, Melville, New York, AIP Conference Proceedings, VOLUME 1051, pp. 25-32. 